\title{
Influência do teor de molibdênio em catalisadores de cobalto e molibdênio suportado em óxido de magnésio para a produção de nanotubos de carbono
}

\author{
Influence of the molybdenum content in cobalt and \\ molybdenum catalysts supported on magnesium \\ oxide for the production of carbon nanotubes
}

Thayana Goulart ${ }^{1}$, Juliana Smarzaro ${ }^{1}$, Laura M. Esteves ${ }^{1}$, Hugo Oliveira ${ }^{1}$

\begin{abstract}
${ }^{1}$ Laboratório de Cinética Reatores e Catálise - RECAT - TEQ/UFF, CEP: 24210-240, Niterói, Rio de Janeiro, RJ, Brasil. e-mail: thayana.goulart@hotmail.com,julianasmarzaro@id.uff.br, lauraesteves@id.uff.br, hugoao@id.uff.br
\end{abstract}

\begin{abstract}
RESUMO
O desenvolvimento dos processos de produção de nanotubos de carbono é amplamente estudado com o objetivo de melhorar a produção, pureza e qualidade do produto. Entre os processos de produção de nanotubos, a deposição química em fase vapor sobre catalisadores é o método mais empregado para produção em larga escala, pois este permite controlar, de modo eficiente, os parâmetros reacionais. Catalisadores de $\mathrm{CoMo} / \mathrm{MgO}$ com baixa concentração de cobalto, $5 \%$ em massa, e três diferentes concentrações de molibdênio, 5, 10 e 20\%, foram preparados pelo método de impregnação seca para estudar o efeito do molibdênio na síntese de nanotubos de carbono. As caracterizações dos catalisadores e dos nanotubos puderam demonstrar as relações entre as propriedades catalíticas e as características dos nanotubos. Os catalisadores com maior concentração de molibdênio apresentaram maior formação de espécies $\mathrm{CoMoO}_{4}$ e $\mathrm{MgMoO}_{4}$ indicando maior interação do cobalto e do suporte com o molibdênio. A elevada concentração de molibdênio nos catalisadores prejudicou a taxa de formação dos nanotubos e a menor grau de grafitização.
\end{abstract}

Palavras-chave: nanotubos de carbono, cobalto, molibdênio, deposição química de vapor.

\begin{abstract}
The development of carbon nanotubes production has been subject of research seeking higher carbon yields and selective processes towards materials with superior purity and quality. Among the methods for nanotube synthesis, the catalytic route is a viable option for large-scale production, since it allows a more effective control of reaction. $\mathrm{CoMo} / \mathrm{MgO}$ catalysts with low loading of cobalt, $5 \mathrm{wt} . \%$, and three different loading of molybdenum, 5, 10 and $20 \mathrm{wt} . \%$, were prepared by incipient wetness impregnation for high yield and pure carbon nanotubes. A detailed characterization of the catalysts and nanotubes indicated a relationship between their properties. Catalysts prepared with higher molybdenum loading showed higher formation of $\mathrm{MgMoO}_{4}$ and $\mathrm{CoMoO}_{4}$ indicating an enhanced interaction of $\mathrm{MgO}$ and cobalt with molybdenum. Excess of molybdenum in the catalyst caused a decrease in carbon yield and produced nanotubes with a lower degree of graphitization.
\end{abstract}

Keywords: carbon nanotube, cobalt, molybdenum, chemical vapor deposition.

\section{INTRODUÇÃO}

Os nanotubos de carbono (CNTs) são estudados intensamente desde a sua descoberta por IJIMA nos anos 90 [1]. Atualmente, grande parte das pesquisas está voltada para a aplicação dos nanotubos de carbono como, por exemplo, na construção de transistores para circuitos eletrônicos, produtos esportivos, fabricação de telas coloridas dobráveis e em biotecnologia [2, 3]. Apesar dos CNTs terem múltiplas utilidades, ainda existe limitações na sua produção com alta seletividade e em escala industrial devido ao alto custo de produção e purificação [4]. 
As três principais técnicas de síntese de CNTs são: descarga por arco, ablação por laser e deposição química de vapor (CVD). Os dois primeiros métodos produzem CNTs com elevada qualidade estrutural, porém as restrições operacionais e, principalmente, os altos custos no processo limitam a sua produção em larga escala $[5,6]$. O método de produção mais utilizado é a CVD que consiste na decomposição de um gás na presença de um catalisador. Este gás deve conter a fonte de carbono para a produção dos nanotubos. O metano é frequentemente usado para a síntese de nanotubos por ser uma fonte abundante e termicamente estável nas condições reacionais. As partículas metálicas do catalisador catalisam a decomposição do gás e, posteriormente, ocorre a deposição de carbono em sua superfície, para que ocorra a formação dos nanotubos é necessário que as partículas metálicas apresentem tamanhos nanométricos [7,8].

Para que a produção de CNTs seja mais eficiente, estuda-se a influência de diferentes tipos de catalisadores em sua síntese. A maioria deles se baseia na utilização de metais de transição ( $\mathrm{Fe}, \mathrm{Ni}, \mathrm{Co}$ ) suportados em óxidos termicamente estáveis (alumina, sílica e óxido de magnésio) [9]. O uso de metais promotores como molibdênio (Mo) ou tungstênio (W) tem melhorado a produção de CNTs, pois a adição dessas espécies causa um efeito estabilizante dos metais de transição em temperaturas elevadas $[9,10]$. AWADALLAH et al. [11] estudaram catalisadores mono e bimetálicos de cobalto e metais do Grupo IV e observaram que o catalisador monometálico $50 \mathrm{Co} / \mathrm{MgO}$ apresentou bom rendimento e estabilidade para a produção de CNTs. Contudo, o catalisador $25 \mathrm{Mo}-25 \mathrm{Co} / \mathrm{MgO}$ apresentou melhor rendimento e estabilidade que o catalisador monometálico e demais catalisadores bimetálicos. Para a síntese de nanotubos de carbono com elevada produção é comum a utilização de catalisadores suportados de cobalto e molibdênio com teor metálico maior que $25 \%$ em massa.

Normalmente, para a aplicação dos CNTs em materiais é necessário fazer um processo de purificação para retirada de impurezas. As principais impurezas encontradas junto aos CNTs são os fragmentos do catalisador (partículas metálicas e o suporte) e outras formas de carbono como o carbono amorfo e grafítico [12]. Para melhorar o grau de pureza do produto, é preciso usar um catalisador seletivo para a formação de nanotubos, evitando-se assim, a produção de outras formas de carbono. Também é importante que o catalisador apresente componentes de fácil solubilização, facilitando a purificação do mesmo. O uso do óxido de magnésio como suporte catalítico pode ser favorável para a etapa de purificação, pois este é facilmente removido por uma solução ácida $[13,14]$. Com isso, este trabalho teve como objetivo verificar o efeito do teor de molibdênio (5, 10 e $20 \%$ em massa) em catalisadores com 5\% de cobalto suportado em óxido de magnésio na produção de CNTs. Os catalisadores foram caracterizados por difração de raios X (DRX), fisissorção de $\mathrm{N}_{2}$ e redução à temperatura programada (TPR). Os CNTs foram caracterizados por DRX, espectroscopia Raman, termogravimetria (TG), microscopia eletrônica de varredura (MEV) e microscopia eletrônica de transmissão (MET).

\section{MATERIAIS E MÉTODOS}

\subsection{Preparação dos catalisares}

Catalisadores bimetálicos com diferentes concentrações de molibdênio, 5, 10 e 20\% em massa, e concentração fixa de cobalto, $5 \%$, nomeados $5 \mathrm{Co}-5 \mathrm{Mo} / \mathrm{MgO}, 5 \mathrm{Co}-10 \mathrm{Mo} / \mathrm{MgO}, 5 \mathrm{Co} 20 \mathrm{Mo} / \mathrm{MgO}$ foram preparados pelo método de impregnação seca com os precursores $\left.\left(\mathrm{NH}_{4}\right)_{6} \mathrm{Mo}_{7} \mathrm{O}_{24}\right) \cdot 4 \mathrm{H}_{2} \mathrm{O}\left(99 \%\right.$ de pureza, Vetec) e $\mathrm{Co}\left(\mathrm{NO}_{3}\right)_{2} \cdot 6 \mathrm{H}_{2} \mathrm{O}(98 \%$ de pureza, Sigma-Aldrich) e suporte de óxido de magnésio (MgO, $97 \%$ de pureza, Merck). Inicialmente, o suporte foi seco a $120^{\circ} \mathrm{C}$ por $12 \mathrm{~h}$ e calcinado a $550{ }^{\circ} \mathrm{C}$, com uma taxa de aquecimento de $5{ }^{\circ} \mathrm{C} \mathrm{min}^{-1}$, por $2 \mathrm{~h}$. Após a coimpregnação do cobalto e molibdênio no suporte, calcinaram-se os catalisadores a $550{ }^{\circ} \mathrm{C}$ com taxa de aquecimento de $5{ }^{\circ} \mathrm{C} \min ^{-1}$ por $2 \mathrm{~h}$.

\subsection{Caracterização dos catalisadores}

As análises de difração de raios X do suporte e dos catalisadores, foram realizadas no equipamento Rigaku Miniflex II utilizando radiação monocromática $\mathrm{CuK} \alpha(\lambda=1,5406 \AA)$, com $2 \theta$ variando de $10^{\circ}$ a $100^{\circ}$ e tempo de contagem de 1 segundo por passo de $0,02^{\circ}$.

A redução a temperatura programada (TPR) foi realizada em um reator de quartzo em forma de U. Antes da análise TPR foi feita uma etapa de secagem de $300 \mathrm{mg}$ de catalisador com a passagem de uma corrente de $\mathrm{He}\left(30 \mathrm{~mL} \mathrm{~min}^{-1}\right)$ de 25 ${ }^{\circ} \mathrm{C}$ até $300{ }^{\circ} \mathrm{C}$ com uma taxa de aquecimento de $10{ }^{\circ} \mathrm{C} \mathrm{min}^{-1}$, após atingida a temperatura de $300{ }^{\circ} \mathrm{C}$, a secagem foi mantida por mais $30 \mathrm{~min}$. Após a secagem, a amostra foi resfriada até a temperatura ambiente e então foi iniciada a etapa de redução química do catalisador com aquecimento até $1000{ }^{\circ} \mathrm{C}$ com passagem de uma mistura gasosa de $5 \% \mathrm{H}_{2} / \mathrm{Ar}\left(30 \mathrm{~mL} \mathrm{~min}{ }^{-1}\right)$ com uma taxa de aquecimento de $10{ }^{\circ} \mathrm{C} \mathrm{min}^{-1}$. Partes dos gases efluentes do reator foram detectados através de um espectrômetro de massas (Pfeiffer, modelo Prisma). A quantidade do consumo de hidrogênio foi obtido de curvas de calibração.

As análises de fisissorção de $\mathrm{N}_{2}$ determinam as áreas específicas (método BET- Brunauer, Emmett e Teller), os diâmetros médios de poros, os volumes de poros e a distribuição de poros (método BJH - Barrett, Joyner e Halenda) do suporte e dos catalisadores. A técnica foi realizada em equipamento Micromeritics ASAP 2020, a uma temperatura criogênica (77 $\mathrm{K})$ através do uso de nitrogênio líquido. Cerca de $1 \mathrm{~g}$ da amostra passou por uma etapa de secagem a $120{ }^{\circ} \mathrm{C}$ por $12 \mathrm{~h}$ na estufa e por um pré-tratamento em equipamento Micromeritics ASAP 2010 sob vácuo a $150^{\circ} \mathrm{C}$ por, aproximadamente, $2 \mathrm{~h}$. 


\subsection{Produção dos nanotubos de carbono}

A produção de nanotubos de carbono foi realizada utilizando $1 \mathrm{~g}$ de catalisador colocado em um reator de quartzo vertical localizado dentro de um forno. Inicialmente reduziram-se os catalisadores com fluxo de $\mathrm{H}_{2}\left(150 \mathrm{~mL} \mathrm{~min}^{-1}\right)$ e sob aquecimento desde a temperatura ambiente até $500{ }^{\circ} \mathrm{C} \mathrm{com} \mathrm{uma} \mathrm{taxa} \mathrm{de} 10{ }^{\circ} \mathrm{C} \mathrm{min}^{-1}$. Em $500{ }^{\circ} \mathrm{C}$, a corrente de $\mathrm{H}_{2}$ foi substituída por uma corrente de $\mathrm{N}_{2}$ e a temperatura continuou aumentando até $800^{\circ} \mathrm{C}$. Em $800{ }^{\circ} \mathrm{C}, \mathrm{N}_{2}$ foi substituído por $\mathrm{CH}_{4}(150 \mathrm{~mL}$ $\mathrm{min}^{-1}$ ), e a reação ocorreu por $30 \mathrm{~min}$ à temperatura de $800{ }^{\circ} \mathrm{C}$. A nomenclatura utilizada para os nanotubos produzidos por cada catalisador foi: CNT_5Co-5Mo/MgO, CNT_5Co-10Mo/MgO, CNT_5Co-20Mo/MgO.

\subsection{Caracterização dos nanotubos de carbono}

As análises de termogravimetria (TG) foram realizadas em um analisador termogravimétrico Shimadzu modelo DTG-60H. Foi utilizada uma atmosfera de $5 \% \mathrm{O}_{2} / \mathrm{He}\left(30 \mathrm{~mL} \mathrm{~min}^{-1}\right)$ e aproximadamente $1,5 \mathrm{mg}$ de amostra foi aquecida da temperatura ambiente até $1000^{\circ} \mathrm{C}$, com uma taxa de aquecimento de $5{ }^{\circ} \mathrm{C} \mathrm{min}^{-1}$. A produção de carbono, $P_{c}$, é dada pela quantidade de carbono depositado na etapa de reação por grama de catalisador e expressa usando a seguinte equação:

$$
\mathrm{P}_{\mathrm{c}}(\%)=\left(\mathrm{m}_{\mathrm{T}}-\mathrm{m}_{\mathrm{Cat}}\right) / \mathrm{m}_{\text {Cat }} \cdot 100
$$

onde $m_{T}$ é a massa total da amostra após a reação de produção de nanotubos de carbono e $m_{\text {Cat }}$ é a massa do catalisador residual após a oxidação dos depósitos de carbono.

As análises de espectroscopia Raman foram feitas em um equipamento Confocal Raman Microscope Alpha 300 da Witec usando uma lente objetiva de 50x e laser com comprimento de onda de $532 \mathrm{~nm}$. As imagens de MEV foram obtidas por um microscópio eletrônico de varredura (JEOL JSM-7100F) e as imagens de MET por um microscópio eletrônico de transmissão (JEOL JEM-1011).

\section{RESULTADOS E DISCUSSÃO}

$\mathrm{Na}$ Figura 1, encontram-se os padrões de difração dos catalisadores (5Co-5Mo/MgO, $5 \mathrm{Co}-10 \mathrm{Mo} / \mathrm{MgO}, 5 \mathrm{Co} 20 \mathrm{Mo} / \mathrm{MgO}$ ) e do suporte. Os principais picos do suporte são referentes ao $\mathrm{MgO}$ (ficha padrão $\mathrm{n}^{\circ}$. 45-0946) fornecida pela JCPDS e estão localizados em $2 \theta=36,9 ; 42,8 ; 62,3 ; 74,7$ e 78, $5^{\circ}$ como observado por ALVARADO et al. [15]. Estes também são os principais picos observados nos difratogramas dos catalisadores. Somente o catalisador $5 \mathrm{Co}-20 \mathrm{Mo} / \mathrm{MgO}$ apresentou dois pequenos picos distintos $\left(2 \theta=23,2\right.$ e $\left.26,4^{\circ}\right)$ que podem ser atribuídos à formação de $\mathrm{MgMoO}_{4}\left(\mathrm{JCPDS} \mathrm{n}^{\circ}\right.$. 722153) ou á formação de $\mathrm{CoMoO}_{4}[16]$ (JCPDS n 21868 ).

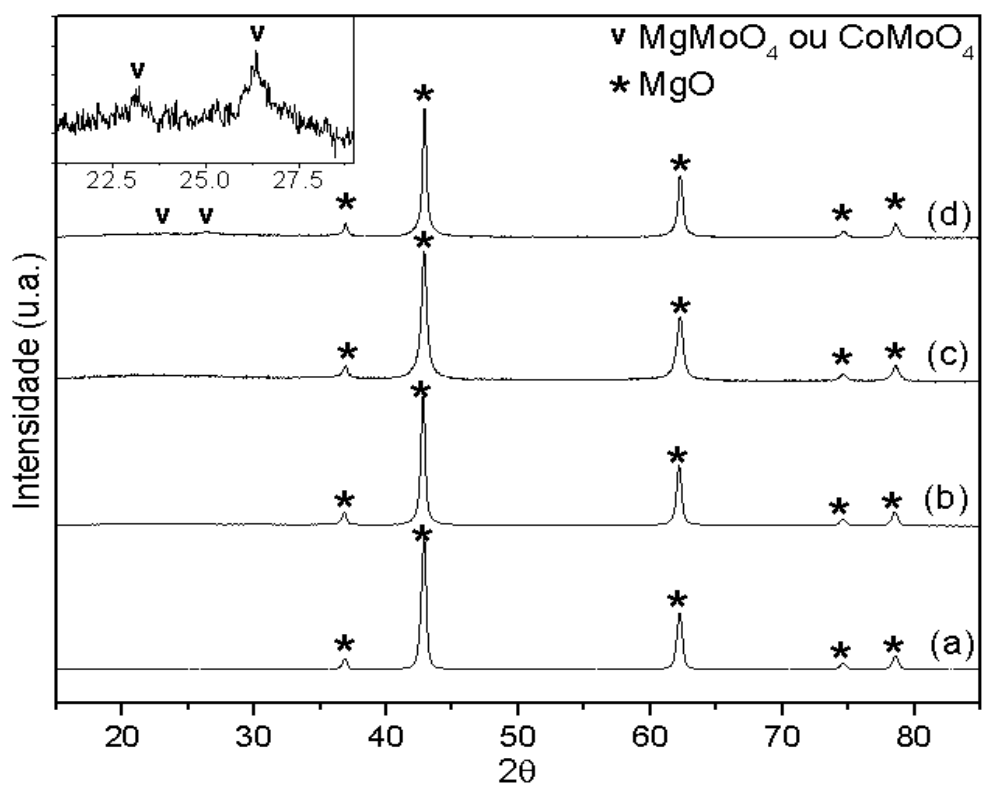

Figura 1: Padrões de difração do suporte e dos catalisadores (a) $\mathrm{MgO}$, (b) $5 \mathrm{Co}-5 \mathrm{Mo} / \mathrm{MgO}$, (c) $5 \mathrm{Co}-10 \mathrm{Mo} / \mathrm{MgO}$, (d) $5 \mathrm{Co}-$ $20 \mathrm{Mo} / \mathrm{MgO}$. 
A posição dos picos do $\mathrm{CoO}(2 \theta=36,5,42,4,61,5,73,5$ e 77,5) (JCPDS no. 65-0902) são muito próximas dos picos do $\mathrm{MgO}$ e, devido a esta proximidade pode ocorrer a sobreposição dos mesmos. Os picos mais intensos da espécie $\mathrm{Co}_{3} \mathrm{O}_{4}$ $\left(2 \theta=19,0\right.$ e $\left.36,9^{\circ}\right)$ (JCPDS no. 43-1003) também apresentam sobreposição com alguns picos de difração das espécies $\mathrm{MgMoO}_{4}, \mathrm{CoMoO}_{4}$ e $\mathrm{MgO}$.

As isotermas do suporte e dos catalisadores estão representadas na Figura 2. As curvas obtidas são isotermas do tipo IV, com histereses tipo H3 associadas ao fenômeno de condensação capilar em estruturas mesoporosas. O aumento do teor de molibdênio nos catalisadores aumentou a quantidade de nitrogênio adsorvido por grama de amostra e intensificou o fenômeno de condensação capilar. Este comportamento provavelmente ocorre devido à destruição da textura original do suporte, $\mathrm{MgO}$, em função da sua hidratação pela utilização de solução aquosa durante a etapa de impregnação, que leva à formação de $\mathrm{Mg}(\mathrm{OH})_{2}$. Desta forma, ao realizar a calcinação dos catalisadores, a transformação do hidróxido em óxido de magnésio em presença do molibdênio pode alterar parte de sua estrutura. Deste modo, a área específica e volume de poros dos catalisadores são alterados.

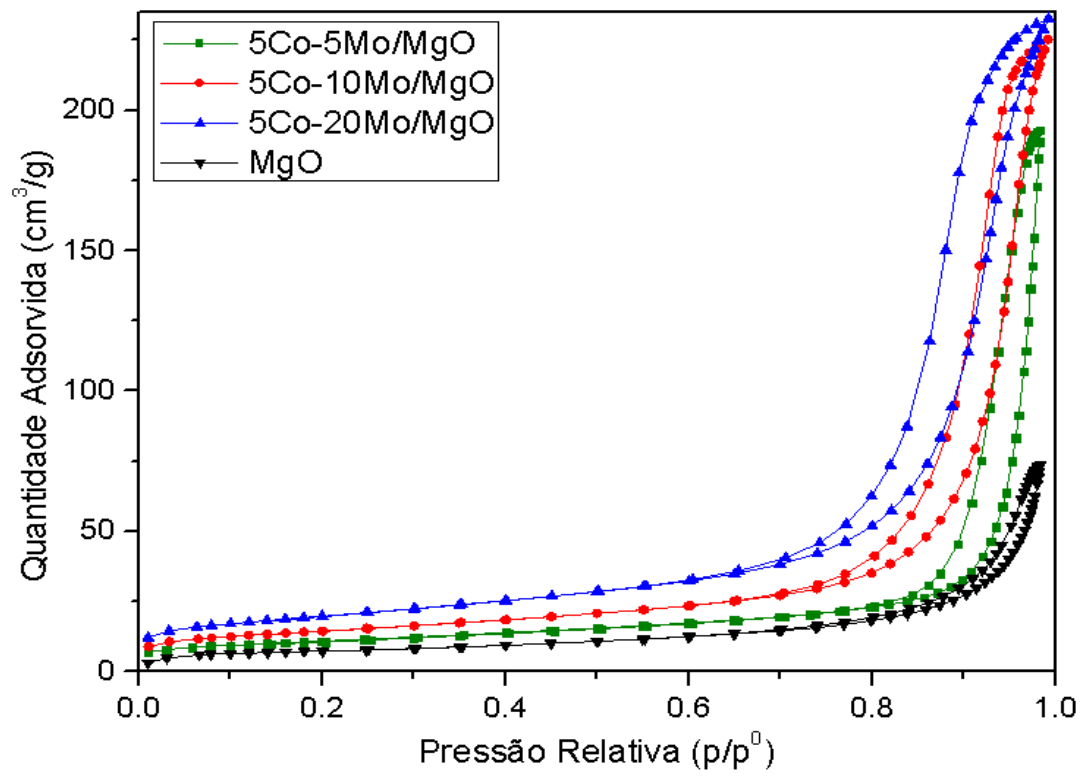

Figura 2: Isotermas de adsorção e dessorção de $\mathrm{N}_{2}$ do suporte e dos catalisadores.

Na Tabela 1 apresentam-se os resultados das áreas específicas e os volumes de poros tanto do suporte como dos catalisadores. Pode-se observar que a área específica e o volume de poros vão aumentando de acordo com o aumento do teor de molibdênio.

Tabela 1: Área específica (ABET) e volume de poros (VP) do suporte e dos catalisadores.

\begin{tabular}{c|c|c}
\hline Amostra & $\mathbf{A}_{\mathrm{BET}} \mathbf{( \mathbf { m } ^ { 2 } / \mathbf { g } )}$ & $\mathbf{V}_{\mathbf{P}} \mathbf{( \mathbf { c m } ^ { \mathbf { 3 } } / \mathbf { g } )}$ \\
\hline $\mathrm{MgO}$ & 27 & 0,12 \\
\hline $5 \mathrm{Co}-5 \mathrm{Mo} / \mathrm{MgO}$ & 38 & 0,29 \\
\hline $5 \mathrm{Co}-10 \mathrm{Mo} / \mathrm{MgO}$ & 52 & 0,35 \\
\hline $5 \mathrm{Co}-20 \mathrm{Mo} / \mathrm{MgO}$ & 71 & 0,36 \\
\hline
\end{tabular}

A técnica de TPR permitiu avaliar os perfis de redução dos catalisadores, os quais encontram-se na Figura 3. O suporte permaneceu inerte durante toda a análise de TPR enquanto os catalisadores apresentaram três picos principais de consumo de hidrogênio. $\mathrm{O}$ primeiro pico entre $350 \mathrm{e} 450^{\circ} \mathrm{C}$ é atribuído à redução de óxidos de cobalto $\left(\mathrm{Co}_{3} \mathrm{O}_{4} \mathrm{e} \mathrm{CoO}\right) \mathrm{com}$ fraca interação com o suporte $[11,17]$. O segundo pico, entre 600 e $750^{\circ} \mathrm{C}$, é característico da redução de óxidos mistos de cobalto e molibdênio $\left(\mathrm{CoMoO}_{\mathrm{x}}\right)$ e óxidos de molibdênio $\left(\mathrm{MoO}_{3}\right)$ [17], e o último pico, entre 850 e $1100{ }^{\circ} \mathrm{C}$, é relativo a redução dos óxidos de cobalto e óxidos de molibdênio com forte interação com o suporte $\left(\mathrm{CoO}-\mathrm{MgO}\right.$ e $\left.\mathrm{MgMoO}_{4}\right)[17$, 18]. $\mathrm{O}$ aumento do consumo de $\mathrm{H}_{2}$ em temperaturas elevadas em função da concentração de molibdênio se dá devido ao aumento de espécies de $\mathrm{Co}$ e Mo que interagem fortemente com o suporte $\left(\mathrm{CoO}-\mathrm{MgO}\right.$ e $\left.\mathrm{MgMoO}_{4}\right)$. 


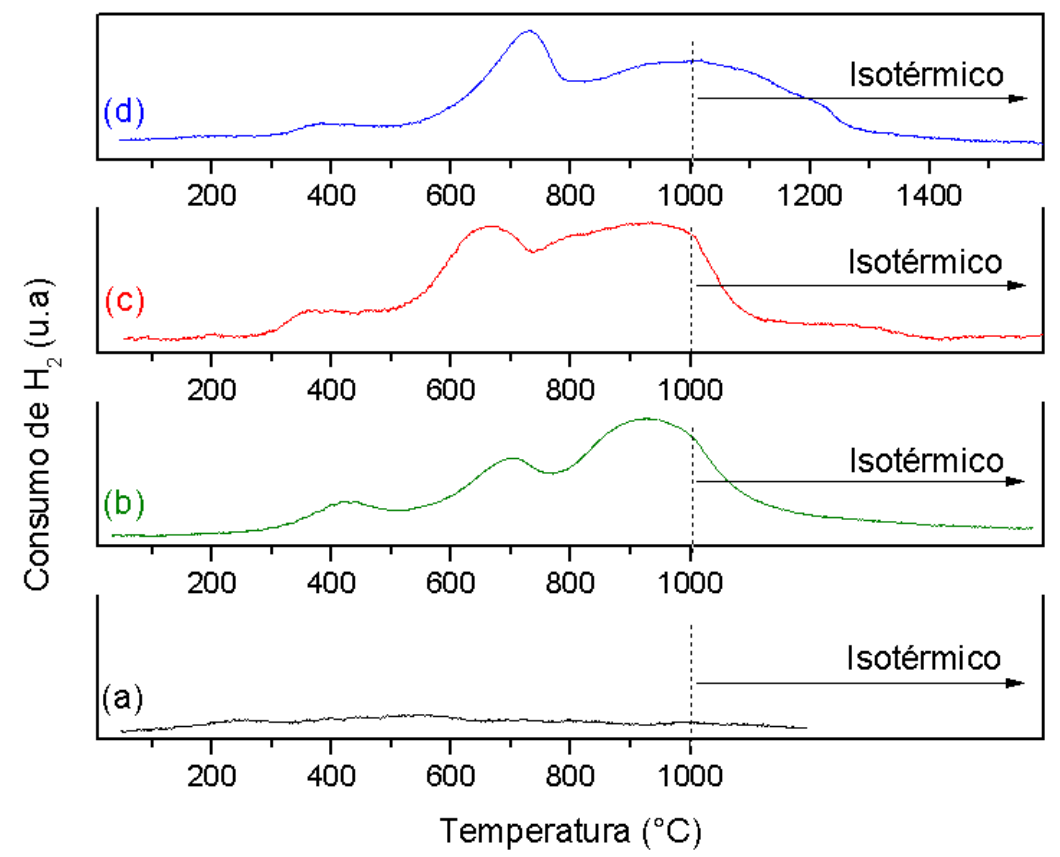

Figura 3: Perfil do TPR do suporte e dos catalisadores (a) $\mathrm{MgO}$, (b) $5 \mathrm{Co}-5 \mathrm{Mo} / \mathrm{MgO}$, (c) $5 \mathrm{Co}-10 \mathrm{Mo} / \mathrm{MgO}$, (d) $5 \mathrm{Co}-20 \mathrm{Mo} /$ $\mathrm{MgO}$.

Os resultados de DRX mostraram que um aumento no teor de molibdênio aumenta a formação das fases $\mathrm{MgMoO}_{4}$ e/ou $\mathrm{CoMoO}_{4}$. Estas duas espécies apresentam padrão de difração semelhante. Outras espécies não foram identificas pela análise de difração. Contudo, é possível observar pelo perfil de TPR dos catalisadores que existem diversas espécies de óxidos diferentes como $\mathrm{CoO}, \mathrm{Co}_{3} \mathrm{O}_{4}, \mathrm{MoO}_{3} \mathrm{CoMoO}_{4}$ e $\mathrm{MgMoO}_{4}$. Não foi possível fazer quantificação destas espécies devido à sobreposição dos perfis de redução. Entretanto, é possível observar que a quantidade das espécies $\mathrm{MoO}_{3} \mathrm{CoMoO}_{4} \mathrm{e}$ $\mathrm{MgMoO}_{4}$, que reduzem em temperatura elevada, aumenta em função do aumento do teor de molibdênio.

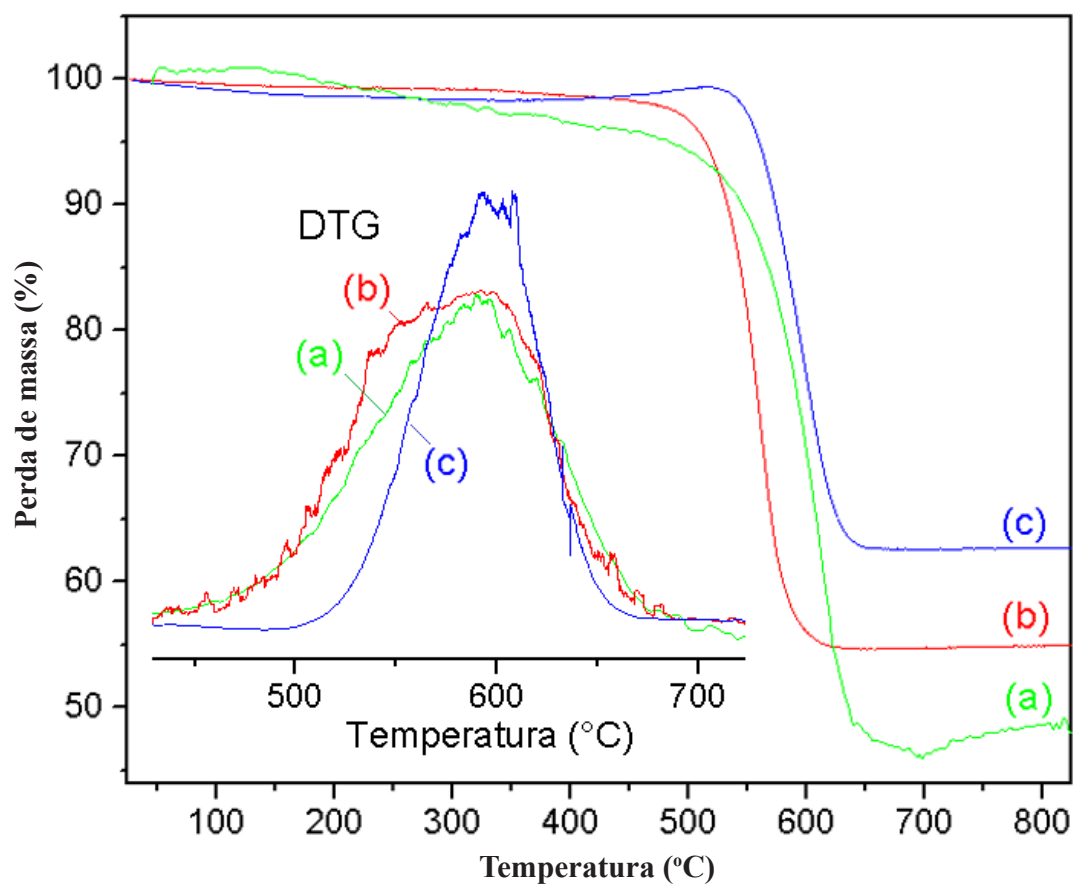

Figura 4: Curvas de perda de massa obtidas por TG dos nanotubos de carbono (a) CNT_5Co-5Mo/MgO, (b) CNT_ $5 \mathrm{Co}-10 \mathrm{Mo} / \mathrm{MgO}$, (c) CNT_5Co-20Mo/MgO. 
A Figura 4 mostra as curvas de perda de massa devido a oxidação dos nanotubos produzidos pelos diferentes catalisadores. Na Figura 4 foi inserido a deriva de primeira ordem das curvas de perda de massa. No inserte é possível observar que a faixa de temperatura de oxidação das diferentes amostras são semelhantes.

A Tabela 2 apresenta os resultados da produção de CNTs e a faixa de temperatura que estes são oxidados. A produção de nanotubos foram calculadas usando a Equação (1) e a faixa de temperatura foi calculada a partir das curvas DTG das amostras. Como a amostra inicial é composta por catalisador e carbono, a perda massa ocorre, principalmente, devido a oxidação do carbono no estado sólido a dióxido de carbono no estado gasoso. Estes valores foram usados para o cálculo da produção de nanotubos. É possível observar na Tabela 2 que um aumento na concentração de molibdênio no catalisador prejudica a produção dos nanotubos.

Tabela 2: Resultado do TG de produção de CNTs em porcentagem e a faixa de temperatura de oxidação dos mesmos.

\begin{tabular}{c|c|c}
\hline CATALSADORES & PRODUÇÃO (\%) & TEMPERATURA ( $\left.{ }^{\circ} \mathbf{C}\right)$ \\
\hline $5 \mathrm{Co}-5 \mathrm{Mo} / \mathrm{MgO}$ & 110 & $470-670$ \\
\hline $5 \mathrm{Co}-10 \mathrm{Mo} / \mathrm{MgO}$ & 81 & $470-670$ \\
\hline $5 \mathrm{Co}-20 \mathrm{Mo} / \mathrm{MgO}$ & 59 & $510-660$ \\
\hline
\end{tabular}

É possível avaliar as estruturas dos CNTs pela razão entre as intensidades da banda D e G observadas por espectroscopia de Raman. Quanto menor for essa razão maior o grau de ordenamento dos CNTs. A banda G está associada a camadas grafíticas e aparece no número de onda de 1500 a $1600 \mathrm{~cm}^{-1}$, já a banda D representa as imperfeições estruturais e é visualizada entre 1200 e $1400 \mathrm{~cm}^{-1}$ [19].

Os espectros Raman dos diferentes CNTs produzidos pelos catalisadores estão representadas na Figura 5. Observa-se que a razão $\mathrm{I}_{\mathrm{D}} / \mathrm{I}_{\mathrm{G}}$ vai aumentando de acordo com o aumento do teor de molibdênio, ou seja, catalisadores com alto teor de molibdênio tendem a desfavorecer a estrutura dos nanotubos.

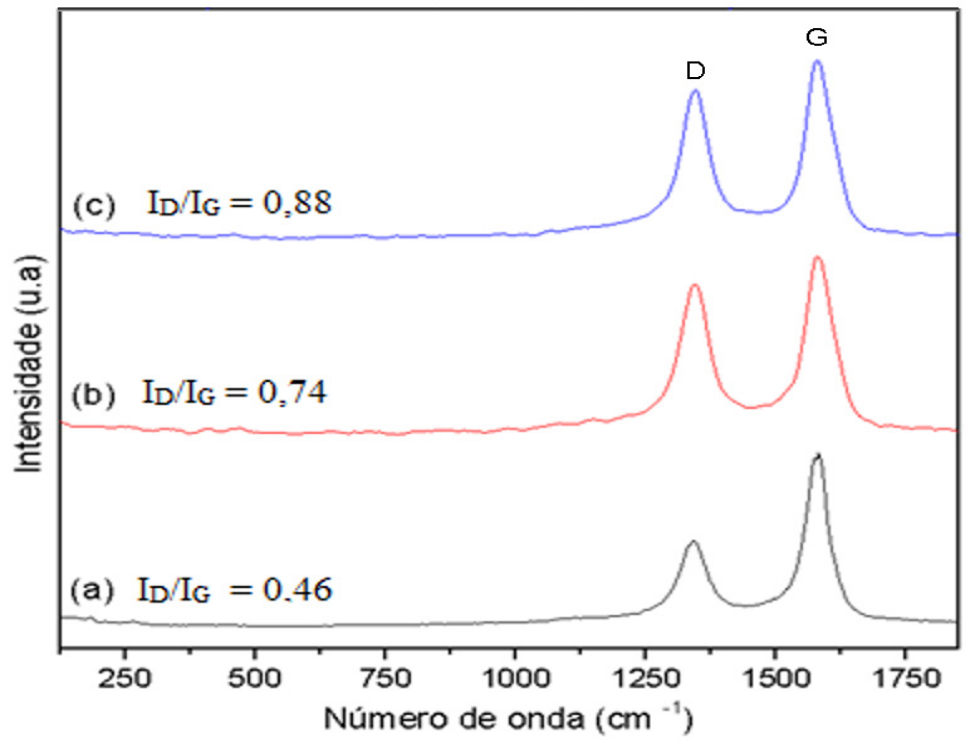

Figura 5: Espectros de Raman (a) CNT_5Co-5Mo/MgO, (b) CNT_5Co-10Mo/MgO, (c) CNT_5Co-20Mo/MgO.

Os catalisadores com menor teor de molibdênio apresentaram maior produção de nanotubos de carbono como pode ser observado pelo resultado de termogravimetria das amostras. KIBRIA et al. [20] apresentaram resultados semelhantes relativos à produção dos nanotubos, onde o catalisador $10 \mathrm{Co}-20 \mathrm{Mo} / \mathrm{MgO}$ apresentou maior produção de nanotubos do que o catalisador com maior fração de molibdênio em sua composição $15 \mathrm{Co}-15 \mathrm{Mo} / \mathrm{MgO}$. YEOH et al. [21] estudaram o efeito da concentração de molibdênio em catalisadores de $5 \mathrm{CoMo} / \mathrm{MgO}$ preparados pelo método sol-gel na produção de nanotubos de carbono por CVD de metano a $800{ }^{\circ} \mathrm{C}$. A concentração de molibdênio variou entre 5 e $40 \%$ em massa e eles encontraram que o aumento do teor de molibdênio também diminui a produção e o grau de grafitização dos nanotubos provavelmente devido a maior formação das espécies $\mathrm{CoMoO}_{4}$ e $\mathrm{MgMoO}_{4}$. Este mesmo efeito também foi observado nos catalisadores deste trabalho, que foram preparados pelo método de impregnação seca. Os resultados de espectroscopia 
Raman e imagens de microscopia eletrônica mostram que os catalisadores com maior teor de molibdênio produziram nanotubos com pior estruturação.

A formação de nanotubos com defeitos estruturais pode ocorrer quando a taxa de decomposição de carbono sobre a superfície do catalisador é maior que a taxa de crescimento dos nanotubos [22]. Um aumento na concentração de molibdênio dos catalisadores pode aumentar a taxa de decomposição dos catalisadores já que molibdênio é ativo para a decomposição de metano [23].

A Figura 6 mostra a imagem de microscopia eletrônica de varredura dos filamentos produzidos sobre o catalisador $5 \mathrm{Co}-10 \mathrm{Mo} / \mathrm{MgO}$. É possível perceber que toda a superfície do catalisador está coberta por filamentos. A Figura 7 é uma imagem obtida por microscopia eletrônica de transmissão e nela é possível observar a presença filamentos com espaços vazios em seu interior e com diâmetro externo de aproximadamente $20 \mathrm{~nm}$. Estas características são típicas de nanotubos com múltiplas paredes.

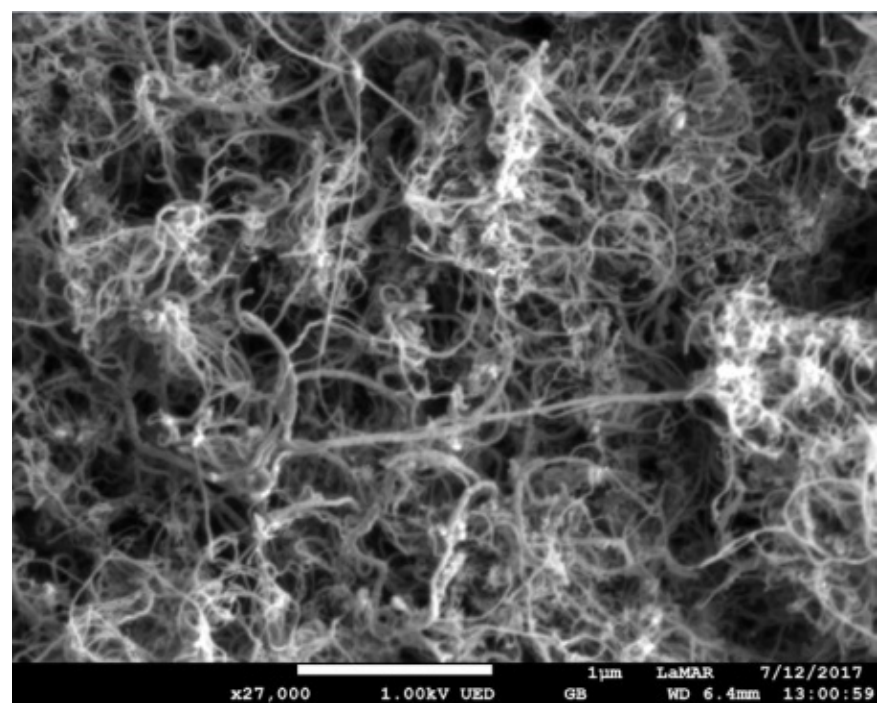

Figura 6: Imagem de MEV dos nanotubos produzidos pelo catalisador 5Co-10Mo/MgO.

Os difratogramas dos nanotubos de carbono produzidos estão representados na Figura 8. Estas amostras não sofreram processos de purificação, desta forma, as amostras são compostas de nanotubos e catalisador. Por isso, além dos picos caraterísticos dos nanotubos de carbono $\left(2 \theta=27^{\circ}\right)$ também foi observado a presença de $\mathrm{MgMoO}_{4}$ ou $\mathrm{CoMoO}_{4}$ no catalisador com $20 \%$ de Mo. Não foi possível identificar a presença de óxidos de cobalto ou outros óxidos de molibdênio.

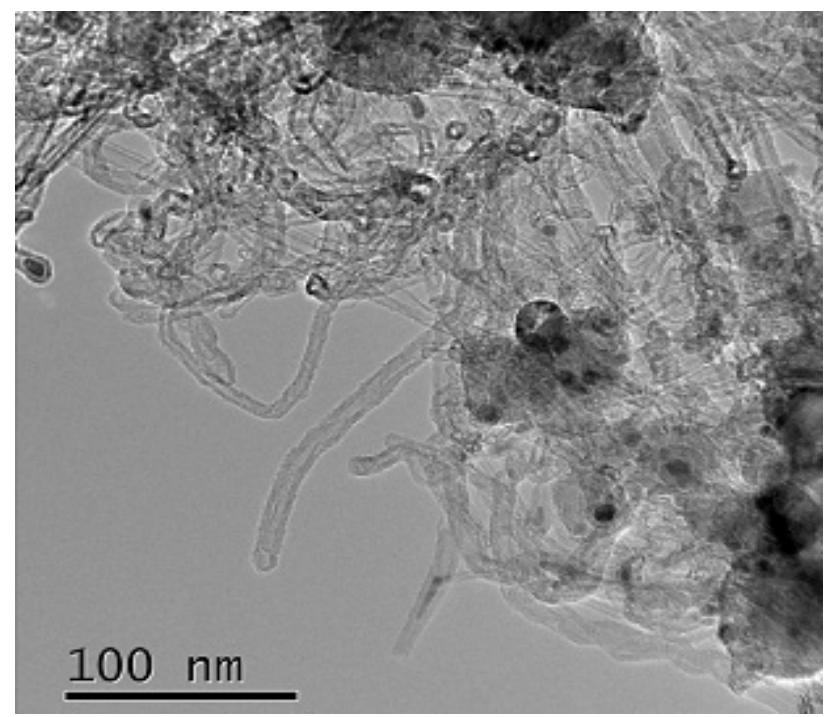

Figura 7: Imagem de MET dos nanotubos produzidos pelo catalisador 5Co-10Mo/MgO. 


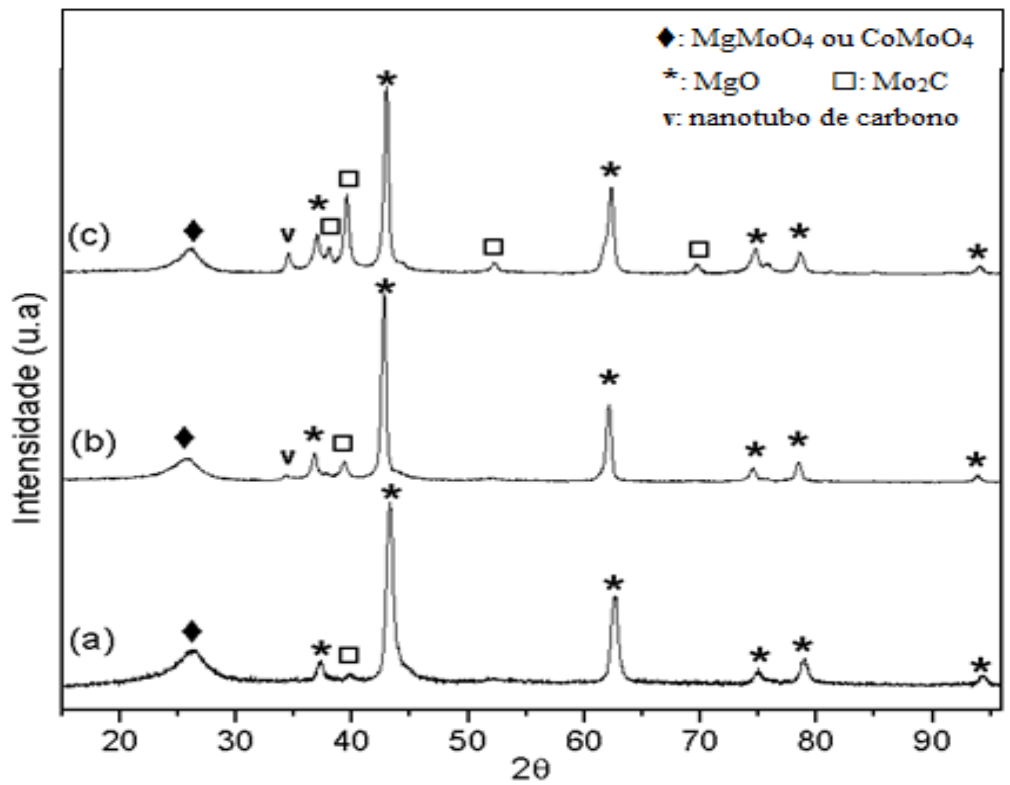

Figura 8: Difratograma dos nanotubos (a) CNT_5Co-5Mo/MgO, (b) CNT_5Co-10Mo/MgO, (c) CNT_5Co-20Mo/MgO.

$\mathrm{O}$ catalisador $5 \mathrm{Co}-5 \mathrm{Mo} / \mathrm{MgO}$ é o mais indicado entre os estudados para produção de nanotubos. Através da técnica de termogravimetria, o mesmo apresentou um único intervalo intenso de perda de massa, sendo assim mais seletivo para produção de nanotubos. A espectroscopia Raman provou que ele apresenta boa estrutura cristalina pois possui a menor razão $I_{D} / I_{G}$. E ele também apresentou maior produção, 1,1g de nanotubos para cada grama de catalisador em 30 min de reação.

\section{CONCLUSÕES}

Os catalisadores com maior teor de molibdênio em sua composição levam a maior formação de espécies como $\mathrm{CoMoO}_{4}$, $\mathrm{MgMoO}_{4}$ e $\mathrm{MoO}_{3}$ e menor formação de $\mathrm{CoO}$ e $\mathrm{Co}_{3} \mathrm{O}_{4}$ durante sua preparação. $\mathrm{O}$ elevado teor de molibdênio diminui fortemente a produção de nanototubos de carbono, de 110 para $59 \%$, e prejudica de maneira significativa a sua estrutura cristalina.

O catalisador que obteve melhor desempenho na produção de nanotubos foi o com menor porcentagem de molibdênio, pois ele apresentou maior produção de nanotubos de carbono, é mais seletivo e apresenta boa estrutura cristalina.

\section{AGRADECIMENTOS}

O presente trabalho foi realizado com apoio da Coordenação de Aperfeiçoamento de Pessoal de Nível Superior - Brasil (CAPES) - Código de Financiamento 001 e ao Programa de Pós-Graduação em Engenharia Química da Universidade Federal Fluminense.

\section{BIBLIOGRAFIA}

[1] IJIMA S.; "Helical microtubules of graphitic carbon” Nature, v. 354, pp. 56-58, 1991.

[2] ALVES, J. O.; ZHUO, C.; LEVENDIS, Y. A.; TENÓRIO, J. A. S.; "Synthesis of carbon nanotubes from sugarcane bagasse”, REM: Revista Escola de Minas, v. 65, pp. 313-318, 2012.

[3] TERRONES, M.; "Carbon nanotubes: synthesis and properties, electronic devices and other emerging applications", International Materials Reviews, v.49, pp. 325-377, 2004.

[4] POPOV, V. "Carbon nanotubes: properties and application”, Material Science Engineering Research, v. 43, 2004, 61102.

[5] JOURDAIN, V.; BICHARA, C.; "Current understanding of the growth of carbon nanotubes in catalytic chemical vapour deposition”,Carbon, v.58, pp. 2-39, 2013. 
[6] SEE, C.; HARRIS, A. “A Review of Carbon Nanotube Synthesis Via Fluidized-Bed Chemical Vapor Deposition”, Industrial \& Engineering Chemistry Research, v. 46, pp. 997-1012, 2007.

[7] NESSIM, G.D. "Properties, synthesis, and growth mechanisms of carbon nanotubes with special focus on thermal chemical vapor deposition”, Nanoscale, pp. 1306-1323, 2010.

[8] ZHAO, J.G.; XING, B.Y.; YANG, H.; et al. "Growth of carbon nanotubes on graphene by chemical vapor deposition", New Carbon Materials, v. 31, pp. 31-36, 2016.

[9] KALUZA, L.; GULKOVÁ, D.; VÍT, Z.; et al. "Effect of support type on the magnitude of synergism and promotion in CoMo sulphide hydrodesulphurisation catalyst”, Appl. Catal., A, v. 324, pp. 30-35, 2007.

[10] NI, L.; KURODA, K.; ZHOU, L.-P.; et al. "Kinetic study of carbon nanotube synthesis over Mo/Co/MgO catalysts." Carbon, v. 44, pp. 2265-2272, 2006.

[11] AWADALLAH, A. E.; ABOUL-ENEIN, A. A.; ABOUL-GHEIT, A. K.; "Impact of group VI metals addition to Co/ $\mathrm{MgO}$ catalyst for non-oxidative decomposition of methane into $\mathrm{CO}_{\mathrm{x}}$-free hydrogen and carbon nanotubes." Fuel, v. 129, pp. 27-36, 2014.

[12] ABDULlAHI, I.; SAKULCHAICHAROEN, N.; HERRERA, J. E., "Selective growth of single-walled carbon nanotubes over $\mathrm{Co}-\mathrm{MgO}$ catalyst by chemical vapor deposition of methane." Diamond and Related Materials, v. 38, pp. 1-8, 2013.

[13] WAN, M. L.; JIA, Y.; FANG, F.; et al. "Synthesis of single- and double-walled carbon nanotubes using the calcined $\mathrm{MgO}$ supported commercial metal oxide as catalysts.” Thin Solid Films, v. 525, pp. 35-39, 2012.

[14] TSOUFIS, T.; XIDAS, P.; JANKOVIC, L.; et al. "Catalytic production of carbon nanotubes over Fe-Ni bimetallic catalysts supported on MgO”, Diamond \& Related Materials, v. 16, pp.155-160, 2007.

[15] ALVARADO, E., TORRES-MARTINEZ, L.M., FUENTES, A.F., et al. "Preparation and characterization of MgO powders obtained from different magnesium salts and the mineral dolomite”, Polyhedron. v. 19, pp.2345-2351, 2000.

[16] RADWAN, N. R.; GHOZZA, A. M.; EL-SHOBAKY, G. A. "Solid-solid interactions in $\mathrm{Co}_{3} \mathrm{O}_{4}-\mathrm{MoO}_{3} / \mathrm{MgO}$ system". Thermochemical Acta, v. 398, pp. 211-221, 2003.

[17] WANG, H. Y.; RUCKENSTEI, E. "Formation of filamentous carbon during methane decomposition over Co-MgO catalysts". Carbon, v. 40, pp. 1911-1917, 2002.

[18] BOER, M. D.; KOCH, E. P. F. M.; BLAAUW, R. J.; et al. "The cobalt-molybdenum interaction in CoMo/SiO 2 catalysts: A CO-oxidation study”, Solid State Ionics, pp. 736-742, 1993.

[19] DRESSELHAUS, M.S.; DRESSELHAUS, G.; SAITO, R.; et al. "Raman spectroscopy of carbon nanotubes"; Physics Reports, v.409, pp. 47-99, 2005.

[20] KIBRIA, A. K. M. F.; SHAJAHAN, M.; MO, Y. H.; KIM, M. J.; et al. "Long activity of Co-Mo/MgO catalyst for the synthesis of carbon nanotubes in large-scale and application feasibility of the grown tubes." Diamond and Related Materials, v. 13, pp. 1865-1872, 2004.

[21] YEOH, W. M.; LEE, K. Y.; CHAI, S.P.; et al. "The role of molybdenum in Co-Mo/MgO for large-scale production of high quality carbon nanotubes", Journal of Alloys and Compounds, v. 493, pp. 539-543, 2010.

[22] SON, S.Y., LEE, Y., WON, S., et al. "High-Quality Multiwalled Carbon Nanotubes from Catalytic Decomposition of Carbon and Materials in Gas-Solid Fluidized Beds", Industrial \& Engineering Chemistry Research, v. 47, n. 7, pp. 2166-2175, 2008.

[23] NI, L., KURODA, K., ZHOU, L.P., et al. "Decomposition of metal carbides as an elementary step of carbon nanotube synthesis" Carbon, v. 47, n. 13, pp.3054-3062, 2009.

\section{ORCID}

Laura Margarida de Matos Martins Esteves

Hugo Alvarenga Oliveira

Juliana Lemos Smarzaro

Thayana Lopes Goulart https://orcid.org/0000-0002-5458-0448

https://orcid.org/0000-0002-1476-0495

https://orcid.org/0000-0002-4958-0101

https://orcid.org/0000-0003-3149-9169 